\title{
Approaches to Rid Cathodic Arc Plasmas of Macro- and Nanoparticles:
}

\author{
A Review \\ André Anders \\ Lawrence Berkeley National Laboratory, \\ University of California, 1 Cyclotron Road, MS 53, Berkeley, CA 94720
}

\begin{abstract}
A major obstacle for the broad application of cathodic arc plasma deposition is the presence of micro- and nanoparticles in the plasma, also often referred to as "macroparticles". This paper reviews the formation of macroparticles at cathode spots, their interaction with the arc plasma and substrate, and macroparticle separation and removal from the plasma by various filtering methods. Nineteen variants of filters are discussed, including Aksenov’s classic $90^{\circ}$-duct filter, filters of open architecture, and the concept of stroboscopic filtering.
\end{abstract}

Keywords:

Filtered Arc, Arc Evaporation, Pulsed, Direct Current (DC), PAPVD. 


\section{Introduction}

Cathodic arc plasma deposition is a coating technology with great potential. Most noticeable, cathodic arc plasmas are fully ionized and can therefore be manipulated with electric and magnetic fields. While electric fields allow for changes in the ion energy and thus structure and properties of the films deposited, magnetic fields are used to guide and homogenize the plasma.

While cathodic arc plasma deposition is a well-established technique for some decorative and protective coatings such as TiN, it is not yet widely used for other materials such as alumina and a-C ("amorphous diamond”). The major obstacle for broad application is the presence of microand nanoparticles in the plasma, also often referred to as “droplets” or "macroparticles”, emphasizing their massive nature compared to ions and electrons. Macroparticle sizes are often quoted to be in the range of $0.1-10 \mu \mathrm{m}$. However, the lower limit is usually determined by the limits of the diagnostic method used, and smaller particles (“nanoparticles”) are abundant [1]. In this paper, we use the term "particle" generically for macroparticles, microparticles, nanoparticles, and droplets.

Particle filters are used to separate and remove particles from the cathodic arc plasma. One important parameter of a filter is its plasma transport efficiency which can be defined as the ratio of the number of ions leaving the filter to the number entering it. However, it is often difficult to determine the ion flux into the filter, and a less ambiguous value is the filtered ion current normalized by the arc current. It is advisable to use this "system coefficient", $\kappa=I_{i, \text { filtered }} / I_{\text {arc }}$, characterizing not only the filter but the coupled arc source and filter system. While the filter's plasma transport efficiency may (hypothetically) reach $100 \%$, the system coefficient is typically 
a few percent. It is important to understand that the system coefficient can never reach $100 \%$ because electrons carry most of the arc current.

Particle generation and interaction with the cathodic arc plasma are discussed first, followed by a compilation of nineteen filter configurations and concepts. The review is completed by discussing particle-substrate interaction. This paper may be seen as a continuation of previous reviews by Boxman and coworkers [2, 3] and by Brown [4].

\section{Particle Generation at Cathode Spots}

The current of cathodic arcs is localized in fragments of minute, non-stationary cathode spots [5]. Spot formation is a necessity to provide sufficient power density for plasma formation, electron emission, and current transport between cathode and anode. Particles originate from the plasmasolid interaction at cathode spots. In his cathode spot model, Jüttner [6] explained particle formation caused by plasma pressure on the melted cathode material (Fig. 1). The production of particles is therefore inherently connected to the existence of non-stationary cathode spots. It cannot be avoided unless the arc is operated in other cathode modes [7, 8] such as the spotless vapor mode (cathode of high vapor pressure material is operated at high temperature [8, 9]), or as an anodic arc (hot, evaporating anode, [8, 10-14]).

Many studies have been performed to determine size and velocity distributions of particles as a function of cathode material and temperature, arc duration and current amplitude, and the presence of external magnetic fields [2, 15-22]. One class of methods analyzes the mass balance

of the cathode which allows one to find specific ion and particle erosion rates (usually expressed in $\mu \mathrm{g} / \mathrm{C}$ ). Other methods are based on the deposition of particles on deposition probes which are 
analyzed by optical and electron-optical instruments. Although these latter methods have practical relevance, they also have a fundamental shortfall: not all particles that arrive at the probe (or substrate) are counted but only those which stick to it.

Since our focus here is on reduction and elimination of particles, we will not review these distribution functions but use only a few relevant data and observations. One of them is that magnetically driven cathode spots produce fewer particles, which can be attributed to a shorter interaction time of the plasma with the cathode material at the spot location. This feature and a better control of the cathode erosion pattern lead to the development of magnetically "steered arcs" [23, 24]. Another observation is that heated cathodes [25] and cathodes of low-melting point material have a high particle erosion rate. This is plausible because the volume of the melted zone between the dense spot plasma and the cathode bulk is relatively large. Finally, we note that most particles are ejected at a shallow angle to the cathode surface, while plasma emission is peaked normal to the surface. This is in agreement with Jüttner's model. A good geometry for a plasma source is one utilizing this "natural” particle-plasma separation.

Kang and co-workers suggested enhancing the natural separation by rotating the cathode at high speeds (4200 r/min). Indeed, they found that most particles can be centrifuged, and plasma deposition with much less particles was demonstrated [26]. However, this method is somewhat impracticable because it is difficult to reliably transfer the arc current to a very fast rotating cathode.

\section{Particle-Plasma Interaction}


Once a particle becomes "airborne", it is subject to intense interaction with the surrounding plasma. It can be considered as a floating (electrically isolated) probe immersed in a streaming, fully ionized plasma. It will be negatively charged with respect to the plasma potential to balance the electric current of the very mobile electrons and less mobile ions [27-29]. Along with the electron and ion currents goes a flux of mass, energy and momentum. Additionally, electrons are thermionically emitted from particles, and mass is lost by evaporation and sputtering. Detailed balances of mass, energy, and momentum have been considered [27, 30, 31]. It has been shown that interaction with the plasma cannot lead to complete particle destruction by evaporation or other mechanisms within the short time a particle flies from the cathode to the substrate [28].

While large particles move along straight trajectories, the negative charge acquired can play a role in the transport of nanoparticles [32]. This effect is discussed later (§ 5).

From a theoretical point of view, momentum transfer from plasma ions ("plasma wind") contributes to the transport of nanoparticles. Simple theoretical estimates show that the "plasma wind" plays a role for small nanoparticles only. Therefore, one has to consider the "bouncing" mechanism for larger particles while a more complex mechanism applies to nanoparticles, including mechanical reflection, electrostatic reflection, and plasma momentum transfer.

\section{Particle Filtering}

\subsection{Straight Magnetic Filter}

For coating applications it is not only the absolute number of particles that is important but the density of particles in relation to the film thickness deposited. In other words: it is the ratio of 
plasma and particles rather than the particle density that is decisive. Straight filters improve the plasma to particle ratio but do not eliminate particles because there is a line-of-sight between cathode and substrate (Fig. 2).

Magnetic filtering in general utilizes plasma guiding by magnetic fields. Plasma guiding is actually accomplished by a combined magnetic field (electrons) and electric field (ions) mechanism. Electrons spiral along magnetic field lines, i.e., their gyration radius is much smaller than the filter size and their collision frequency is smaller than the gyration frequency: they are said to be magnetized. In contrast, ions are not magnetized because their gyration radius is greater than the characteristic filter size (usually the inner filter radius). Although not magnetized, ions follow the guided electrons electrostatically so as to keep the plasma quasineutral.

The goal of filter research is to increase the plasma transport efficiency through the filter and to reduce the residual particle flux to the substrate location. The mechanism of plasma transport is the subject of a number of papers, see, for instance, [33-41].

Plasma is magnetically guided to the substrate thus enhancing the plasma density and deposition rate as compared to free plasma expansion. Only a small fraction of particles can reach the substrate, the others are caught or reflected by baffles and shields. Straight filters have generally a higher plasma transmission than curved filters, and particle reduction achieved may be adequate for some coating applications. Plasma density in straight filters decays exponentially with attenuation lengths typically between 10 and $25 \mathrm{~cm}$ [37, 42]. 


\subsection{Straight Magnetic Filter Combined with Annular-Cathode Plasma Source}

The biggest drawback of straight filters is that there is a line-of-sight between cathode and substrate, and plenty of particles can still be found on the substrate surface. Treglio [43] proposed the use of a cathodic arc plasma source with an annular cathode (Fig. 3). If combined with a straight duct (or short annular magnetic field coil), no line of sight exist. No data have been published on the maximum plasma transport achieved.

\subsection{The Classic $90^{\circ}$ Duct Filter}

Many attempts have been made to completely eliminate particles by placing the cathode out-ofsight from the substrate. Most often, the plasma is magnetically guided in curved filters. Plasma electrons and ions are spatially separated from particles based on the huge difference in chargeto-mass ratio. While the plasma stream can easily be bent, the relatively massive particles move along almost straight trajectories. Aksenov and co-workers introduced a 90-duct filter [33, 44], schematically shown in Fig. 4. The duct filter consists of a curved tube (the "duct") which is surrounded by magnetic field coils generating a curved axial field; the $90^{\circ}$ duct can also be considered as a quarter magnetic torus. To reduce particle transport by multiple reflections, Aksenov and co-workers inserted baffles in their duct. They found that biasing the duct positively improves plasma transport and filter efficiency. This type of filter has been widely used in cathodic arc R\&D over the last decade, see e.g., [4, 45-49]. While Aksenov and coworkers developed a relatively large system (duct length about $1 \mathrm{~m}$ ) for DC plasma operation, this equipment can easily be scaled. Small systems suitable for pulsed arc operation (duct length about $10 \mathrm{~cm}$ ) show the same principal features. 
Storer et al. [37] found that the plasma transport efficiency decreases exponentially with the length of the filter, and the curved filter has a shorter attenuation length than straight filters of comparable construction and size. Plasma transport through $90^{\circ}$ ducts was also the subject of more detailed experimental investigations [34, 50, 51]. For good plasma transport, the duct must be biased with respect to the plasma, typically to about +10 to $+25 \mathrm{~V}$, an effect already noted in the pioneering work of Aksenov and coworkers [33]. This feature was recognized also by Bilek and coworkers [39, 52], who showed that applying a positive bias to a strip electrode that is located near the outer wall of the interior of the duct produces a similar effect. With optimized bias (about $+20 \mathrm{~V}$ ) and at relatively strong magnetic fields (of order $100 \mathrm{mT}$ ), system coefficients of $90^{\circ}$ duct filters as high as $2.5 \%$ have been found [34].

\subsection{The Knee-Filter}

Because plasma losses become greater for larger bent angle of the filter, it is reasonable to design a non-line-of-sight filter which has a relatively small bending angle and is otherwise straight. This concept has been realized by Sanders and Falabella at LLNL with their Knee-Filter (Fig. 5), now commercially available from Commonwealth Scientific Corporation [53]. As in the classic $90^{\circ}$-duct filter, particle reflection is reduced by baffles inserted in the two straight duct sections. The commercial Knee-Filter is designed for DC operation.

\subsection{Filter Segments for Pulsed High-Current-Arc (HCA)}

Deposition with pulsed arcs can have advantages over DC operation. For instance, the power consumption can be easily regulated via the arc duty cycle (on/off ratio) rather than the arc 
current (low arc currents lead to the familiar chopping phenomenon). Spot steering is not an issue. Pulse arcs can be operated with high currents, matching the average plasma production of DC arcs. The plasma transport of pulsed high-current arcs (2 kA) was investigated using a duct filter similar to the classic $90^{\circ}$ filter. The ion current at the filter exit was very high: up to $7 \%$ of the arc current [54]. The High-Current Arc (HCA) system developed at the Fraunhofer Institute [55] operates with very high currents (peak $5 \mathrm{kA}$ ) and arc repetition frequency (up to $300 \mathrm{~Hz}$ ). The average plasma production can exceed even typical DC values (arc currents of order $100 \mathrm{~A}$ ). Although the arc is pulsed, the magnetic field is DC-powered and configured in $30^{\circ}$-duct segments which may be combined to $30^{\circ}, 60^{\circ}, 90^{\circ}$, ect. particle filters (Fig. 6).

\section{6. Rectangular $90^{\circ}$ Duct Filter}

Early versions of a rectangular $90^{\circ}$ duct filter were developed in the 1980s in the Soviet Union as part of the Cold War weapons and technology effort (Secret Soviet Author Certificate, unpublished [56]). By using very elongated rectangular filters, the possibilities of particle removal are expanded toward industrially relevant large-area coatings. Welty [57] developed a patented $90^{\circ}$-duct filter with a rectangular cross section (Fig. 7). Interestingly, this filter utilizes also magnets other than solenoids. These magnets, either electromagnets or permanent magnets, are located outside the duct wall. The shape and cross section of a rectangular duct filter is matched to the size of the rectangular cathode, with one of its dimensions much longer than the other. The spot can be magnetically steered back and forth along the cathode. Very large-area coatings are possible when the substrate is moved perpendicular to the long extension of the filter. 


\subsection{Dome Filter}

The idea of the Dome Filter is to insert a line-of-sight blocking shield between cathode and substrate and to guide the plasma round it with a dome-shaped magnetic field [58, 59] (Fig. 8). In contrast to all previous filters, no duct (tube) is involved but source and substrate are located in the same processing chamber. The central shield can also serve as the anode. A very high source-filter system coefficient of $3.5 \%$ is claimed.

\subsection{Bi-directional Linear Filter}

While the original Dome Filter has a cylindrical cathode with the plasma generated in an axially symmetric fashion, the geometry can be modified in such a way as to obtain two "linear" plasma flows streaming in opposite directions [60]. The basic geometry of a "bi-directional linear arc and filter" is shown in Fig. 9. It can be understood as an "elongated” or "linear” Dome Filter, rotated by $90^{\circ}$ when compared to Fig. 8 . In contrast to the dome filter, the plasma streams along both directions of the magnetic field lines. Also here, the arc spots are magnetically driven along the cathode periphery. The longer the cathode the smaller the plasma losses at the ends of the cathode bar. The setup is suitable as an axial source in a batch system or as a source for a linear dual substrate system.

\subsection{Radial Filter}

Aksenov and coworkers have described a novel kind of geometry involving an axially symmetric magnetic filter configuration [61]. Deposition occurs on the inside of a substrate holder of large diameter (Fig. 10). Both mirror and ring-cusp magnetic geometries (adding and opposing 
magnetic field coils, respectively) were investigated. Good efficiency and high film deposition rates were demonstrated $\left(10 \mu \mathrm{m} / \mathrm{h}\right.$ of TiN over $0.2 \mathrm{~m}^{2}$ substrate area).

\subsection{Annular Cathode Apparatus}

In their search for alternative geometries and principles to the classic $90^{\circ}$ filter duct, the Ukrainian researchers Kljuchkov et al. positioned substrates inside annular cathodes and guided the plasma magnetically to the substrates [62]. Fig. 11 shows the setup with two cathodes. The substrates surface does not face the annular cathode surface; plasma streams to the enclosure which serves as the anode. The magnetic field prevents some of the plasma from arriving at the anode; part of the plasma is deposited onto the substrate. This geometry does not prevent particles from being reflected onto the substrate. No data are available on the quality of coating and system efficiency coefficient. This geometry also limits the substrate to certain shapes and sizes.

\subsection{Nested Venetian-Blind Filter}

In another step from the dome filter, Ryabchikov and co-workers made the blocking shield partially transparent to the plasma by designing the shield as a concentric, nested venetian blind (Fig. 12) [63, 64]. A reduction of two orders of magnitude in the particle fraction has been reported. The plasma transport efficiency is claimed to be up to 70\% (system coefficient about 7\%), but this number needs to be confirmed.

\subsection{Linear Venetian-Blind Filter}


Ryabchikov also proposed and built a linear version of a Venetian Blind filter, however, few details were provided in his paper [65]. The linear version has the advantage that it can be scaled for large area-coatings, similar to the rectangular $90^{\circ}$-duct filter. Based on Ryabchikov's design, a linear Venetian Blind filter was built and tested at LBNL (Fig. 13) [66]. It was found that the filter efficiency dramatically increases with the current through the filter vanes which generates the magnetic field. Under optimum conditions (filter current greater than $1 \mathrm{kA}$ ), the system coefficient was found to be as high as $2.5 \%$.

\subsection{Freestanding $90^{\circ}$ Filter}

Although the classic $90^{\circ}$-duct filter is most widely used, the problem of particle reflection or "bouncing" is insufficiently addressed. There is still a non-negligible probability that a particle is transmitted through the filter although there is no direct line-of-sight. Freestanding or openarchitecture filters improve the situation (Fig. 14). There is no duct present, and the magnetic field is produced by only a few turns of a field coil. The current through the filter coil must be high to obtain a magnetic field of, say, $10 \mathrm{mT}$ or more. Often the arc current is conveniently used for this purpose $[37,67,68]$. Either particles may leave the filter through openings between the turns of the field coil, or they may stick to the turns. Unfortunately, there is still a small but non-zero probability that a particle will be reflected from a turn of the coil exactly in the direction of the substrate, and therefore even freestanding filters do not guarantee completely particle-free film deposition.

\subsection{S-Duct Filter}


In an effort to further reduce the flux of residual particles, filters have been developed whose bend angle exceeds $90^{\circ}$. For instance, one could continue to bend the filter to $180^{\circ}$ or any other large angle [69]. Plasma losses in such filters become severe, and these filters seem to be practicable for ultrathin-film coating applications only. It is known that the plasma stream in a $90^{\circ}$ filter is offset from the filter axis, possibly caused by several reasons such as asymmetry in the magnetic field structure, plasma drift in crossed $\mathbf{E}$ and $\mathbf{B}$ fields, and action of the centrifugal force. In any case, rather than increasing the angle beyond $90^{\circ}$, one may offset these shifts by bending the plasma back into an S-shaped filter (Fig.15). This has been done with some success, and a system coefficient of $0.6 \%$ has been reported [70]. The residual particle flux to the substrate was reduced down to the limits of detection.

\subsection{Freestanding S-Filter}

With the same argument which leads from a classic $90^{\circ}$-duct filter to a freestanding $90^{\circ}$ filter, a freestanding S-filter (i.e., without duct) can also be developed. Such a filter is an excellent research tool because it allows one to easily insert deposition probes [71] for monitoring the plasma position and density as a function of various parameters. Figure 16 shows a freestanding S-filter with extremely well collimated plasma, illustrating magnetic guiding of the plasma.

\subsection{Twist Filter}

A new filter is currently under development which has been dubbed "Twist Filter". It can be understood as a derivative of the free-standing S-filter, with the curvature of bent being in three dimensions: the "S" is twisted out of plane. This has the advantage that particles entering the 
filter at an angle with respect to the entrance axis cannot be transmitted without multiple scattering. More data can be expected in the near future.

\subsection{Electrostatic Filter}

While the previous filters employ a combined magnetic (electrons) and electric (ions) transport mechanism, Meyer and Scheibe recently described an electrostatic filter for laser-triggered pulsed cathodic arcs [72]. The basic configuration is shown in Fig. 17. Although only few details have been disclosed and described, it is clear that without magnetic field, a large crosscurrent must flow in order to maintain a potential difference between the two guiding electrostatic shields. In fact, the current may exceed the arc current, and the ohmic heat load on the shields would be excessive if the cross-current were continuous. Since the are is pulsed, the heat issue is mitigated by the duty factor.

\subsection{Stroboscopic Filter}

A radically different approach compared to all previous filters is realized in a Stroboscopic Filter. While previous filters try to spatially separate particles and plasma, stroboscopic filtering utilizes the different drift velocities of plasma and particles to separate them temporally and spatially. By combining time-of-flight (TOF) separation with a stroboscopic shutter, plasma and particles can be separated. While conventional filtering can be pulsed or DC, stroboscopic filtering is always pulsed. The principal setup is shown in Fig. 18. To reduce plasma losses, the TOF length is enclosed by a magnetic field coil, similar to a straight duct. Although a stroboscopic filter has not yet been built, one can easily determine the required TOF length and shutter speed if the particle velocity distribution is known. Measurements of carbon particle 
velocities have been recently done [73]. One can easily show that the very slow particles present a possible issue since they may be still present when the shutter opens for the next discharge plasma.

\subsection{Rotating Blade Filter}

In the early 1970's Utsumi and English [21] developed a rotating blade filter that they used to measure particle velocities for $\mathrm{Au}, \mathrm{Pd}$, and $\mathrm{Mg}$ arcs. In contrast to a stroboscopic filter, the rotating blade filter can operate with DC arcs. The rotating blade filter, Fig. 19, is the only filter that has a line-of sight between cathode and substrate and still avoids particle deposition (with the exception of "bounced" particles). The principle of operation is obvious from Fig. 19: if the blades rotate fast enough, particles are deflected on their way to the substrate by the fast moving blades. Utsumi and English operated this filter with rotational speeds up to $3100 \mathrm{r} / \mathrm{min}$, eliminating all particles with velocities less than $300 \mathrm{~m} / \mathrm{s}$.

\section{Particle-Substrate Interaction}

As mentioned earlier, not all particles arriving at the substrate will stick to it. In fact, it is likely that particles which make it through the filter by reflections will also be reflected from the substrate. Even if they don't stick, it is conceivable that their impact may cause damage to the growing film. The particles which do stick will certainly degrade the quality of the coatings.

One may further reduce the sticking probability if additional forces act on the particle when it is near or at the substrate surface. One of these forces could be the centrifugal force which occurs when the substrate is rapidly rotated during deposition. In Fig. 20 one can clearly see the bright 
traces of particles impacting a rotating disk. One can also see reflected particles, including their images mirrored from the smooth disk surface. The disk axis is slightly out of alignment, causing the images to appear modulated (this can be used to determine the particle's velocity). We can see that particles are slower after reflection, and many carbon particles fracture into several pieces. Figs. 21 shows carbon particles sticking to a disk which was spinning. The particle density at the outer rim (bottom, high centrifugal force) is less than at the inner rim (top, small centrifugal force). However it is obvious that this approach is not suitable for complete particle removal.

Since particles are negatively charged by the interaction with the surrounding plasma, an electric force may also influence the particle interaction with the substrate. Keidar and co-workers [74, 75] predicted and detected a reduction of particles when the substrate was negatively biased. This effect was confirmed in a recent, extended study by Aharonov and Keidar [76]. Yin and McKenzie [77] deposited $\mathrm{CuCr}$ on negatively biased silicon wafers which were aligned with the flux of plasma exiting a $90^{\circ}$-duct filter (Fig. 22). They have shown that particle deposition can be dramatically reduced this way.

\section{Conclusions and Outlook}

Although particle filtering has been introduced more than two decades ago, much insight has been gained over the last years in plasma and particle transport mechanisms, and numerous novel approaches have been proposed and tested. Plasma losses in filters have been reduced, and plasma transport efficiencies of order 50\% have been achieved in modern filters (corresponding to system coefficients of about 5\%), while reducing particle transmission down to the detection 
limit. Filter sizes range from a few $\mathrm{cm}$ up to meters. Pulsed systems can compete with the more traditional DC systems when operated at arc high current and duty cycle. Further improvements in terms of residual particle reduction can be anticipated by implementing sophisticated baffle structures which are well adapted to the geometry of the source, including its magnetic field. Simulation and design of the guiding field structure plays an increasing role, including the coupling of source and filter. New concepts and designs are under development, including twistfilter, electrostatic filter, and a stroboscopic filter.

\section{Acknowledgements}

I am thankful for numerous stimulating discussions with many colleagues, including Ian Brown, Robert MacGill, Michael Dickinson, Othon Monteiro, Thomas Schülke, Michael Keidar, and Marcela Bilek. This work was supported by the Advanced Energy Projects and Technology Research Division, U.S. Department of Energy, under Contract No. DE-AC03-76SF00098. 


\section{Figure Captions}

Fig. 1 Particle formation at cathode spots: a pool of liquid cathode material (black) is pushed by spot plasma.

Fig. 2 Schematic of a plasma source with straight filter.

Fig. 3 Cathodic arc plasma source with annular cathode, coupled to a short straight filter. The thin lines indicate the annular region of line-of-sight.

Fig. 4 Schematic of the classic $90^{\circ}$-duct filter.

Fig. 5 Schematic of the $45^{\circ}$-Knee-Filter.

Fig. 6 Setup of the High-Current Arc (HCA) with straight and $30^{\circ}$-filter segments.

Fig. 7 Schematic of a rectangular $90^{\circ}$-filter; large area processing is possible by moving the substrate perpendicular to the long duct opening.

Fig. 8 Schematic of a Dome Filter. The particle shield can also serve as the anode. The cathode spot rotates on the surface on the cylindrical cathode.

Fig. 9 Schematic of a linear bi-directional arc source and filter; the anode could be the chamber, and the field-generating coils or permanent magnets are not shown. Plasma follows both directions of the magnetic field lines.

Fig. 10 Schematic of the Radial Filter, here with 2 arc sources. In principal, more sources can be radially arranged, and the substrates can be placed 360 degrees azimuthally.

Fig. 11 Annular cathode coating apparatus with the substrate located inside the cathodes.

Fig. 12 Schematic of a nested, concentric Venetian-Blind Filter. Filter current of up to $1 \mathrm{kA}$ is flowing through the nested filter rings (i.e., vanes or baffles), generating the magnetic field which guides the plasma through the openings. 
Fig. 13 Schematic of a Linear Venetian-Blind Filter, working with the same principle as the nested, concentric version. The loops indicate the current connections between individual vanes.

Fig. 14 Freestanding $90^{\circ}$ filter, operating with the arc current (200 A). Platinum plasma. Filter dimensions: minor radius $3 \mathrm{~cm}$, major radius $15 \mathrm{~cm}$.

Fig. 15 Schematic of an S-duct filter.

Fig. 16 Freestanding S-filter, operating with a pulsed high arc current (1 kA). Titanium plasma. Filter dimensions: minor radius $2.8 \mathrm{~cm}$, major radius $5.3 \mathrm{~cm}$.

Fig. 17 Electrostatic filter for laser-triggered pulsed arcs.

Fig. 18 Concept of a Stroboscopic Filter.

Fig. 19 Rotating Blade Filter: Plasma and particles are emitted from a cathode that is located inside a fast rotating blade system.

Fig. 20 Disk rotating with $8000 \mathrm{r} / \mathrm{min}$ during deposition of carbon from an unfiltered cathodic arc plasma source. Picture taken with a CCD camera; exposure time $16.7 \mathrm{~ms}$.

Fig. 21 Dark-field micrographs of the hard disk shown in Fig. 15. Top: section of inner rim, Bottom: section of outer rim.

Fig. 22 Deposition on negatively biased substrates aligned with the plasma flux exiting a $90^{\circ} \mathrm{duct}$ filter. 


\section{References}

[1] O. R. Monteiro and A. Anders, "Vacuum-arc generated macroparticles in the nanometer range,” presented at XVIIIth Int. Symp. on Discharges and Electrical Insulation in Vacuum, Eindhoven, The Netherlands, 1998, 593-596.

[2] R. L. Boxman and S. Goldsmith, Surf. Coat. Technol. 52 (1992) 39-50.

[3] R. L. Boxman, V. Zhitomirsky, B. Alterkop, E. Gidalevich, I. Beilis, M. Keidar, and S. Goldsmith, Surf. Coat. Technol. 87-88 (1996) 263-270.

[4] I. G. Brown, "Cathodic Arc Deposition of Films," in Annual Review of Materials Science, vol. 28. Palo Alto, CA: Annual Reviews, Inc., 1998, pp. 243-269.

[5] B. Jüttner, V. F. Puchkarev, E. Hantzsche, and I. Beilis, "Cathode Spots," in Handbook of Vacuum Arc Science and Technology, R. L. Boxman, P. J. Martin, and D. M. Sanders, Eds. Park Ridge: Noyes, 1995, pp. 73-281.

[6] B. Jüttner, Beitr. Plasmaphys. 19 (1979) 25-48.

[7] S. Anders and A. Anders, IEEE Trans. Plasma Sci. 19 (1991) 20-24.

[8] V. I. Gorokhovsky, V. P. Polistchook, I. M. Yartsev, and J. W. Glaser, "Distributed Arc Sources,” in Handbook of Vacuum Arc Science and Technology, R. L. Boxman, P. J. Martin, and D. M. Sanders, Eds. Park Ridge, NJ: Noyes Publications, 1995, pp. 423-444.

[9] A. I. Vasin, A. M. Dorodnov, and V. A. Petrosov, Sov. Tech. Phys. Lett. 5 (1979) 634636.

[10] A. M. Dorodnov, A. N. Kuznetsov, and V. A. Petrosov, Pis'ma Zh. Tekhn. Fiz. 5 (1979) $1101-1106$.

[11] H. Ehrich, B. Hasse, M. Mausbach, and K. G. Müller, IEEE Trans. Plasma Sci. 18 (1990) 895-903. 
[12] H. Ehrich, B. Hasse, M. Mausbach, and K. G. Müller, J. Vac. Sci. \& Technol. A 8 (1990) 2160-2164.

[13] H. Ehrich, J. Schuhmann, G. Musa, A. Popescu, and I. Mustata, Thin Solid Films 333 (1998) 95-102.

[14] S. Meassick, C. Chan, and R. Allen, Surf. \& Coat. Technol. 54 (1992) 343-348.

[15] W. D. Davis and H. C. Miller, J. Appl. Phys. 40 (1969) 2212-2221.

[16] J. E. Daalder, J. Phys. D: Appl. Phys. 9 (1976) 2379-2395.

[17] S. Anders, A. Anders, K. M. Yu, X. Y. Yao, and I. G. Brown, IEEE Trans. Plasma Sci. 21 (1993) 440-446.

[18] M. Kandah and J.-L. Meunier, J. Vac. Sci. Technol. A 13 (1995) 2444-2450.

[19] M. Kandah and J. L. Meunier, IEEE Transactions on Plasma Science 24 (1996) 523-527.

[20] S. Shalev, R. L. Boxmann, and S. J. Goldsmith, J. Appl. Phys. 58 (1985) 2503-2507.

[21] T. Utsumi and J. H. English, J. Appl. Phys. 46 (1975) 126-131.

[22] S. Shalev, S. Goldsmith, R. L. Boxman, S. Einav, and J. M. Avidor, J. Phys. E: Sci. Instrum. 17 (1984) 56-61.

[23] S. Falabella and D. A. Karpov, "Continous cathodic arc sources," in Handbook of Vacuum Science and Technology, R. L. Boxman, P. J. Martin, and D. M. Sanders, Eds. Park Ridge: Noyes, 1995, pp. 397-412.

[24] S. Ramalingam, C. B. Qi, and K. Kim, Controlled vacuum arc material deposition, method and apparatus, Patent US 4,673,477, June 16, 1987.

[25] B. Jüttner, Physica 114C (1982) 155-261.

[26] G. H. Kang, H. Uchida, and E. S. Koh, Surf. Coat. Technol. 68/69 (1994) 141-145.

[27] R. L. Boxman and S. Goldsmith, J. Appl. Phys. 52 (1981) 151-161. 
[28] A. Anders, J. Appl. Phys. 82 (1997) 3679-3688.

[29] I. I. Beilis, M. Keidar, R. L. Boxman, and S. Goldsmith, IEEE Trans. Plasma Sci. 25 (1997) 346-352.

[30] X. Chen, J. Chen, and Y. Wang, J. Phys. D: Appl. Phys. 27 (1994) 1637-1645.

[31] P. Froebrich, Annalen der Physik 6 (1997) 403-21.

[32] M. Keidar, I. I. Beilis, R. L. Boxman, and S. Goldsmith, IEEE Trans. Plasma Sci. 24 (1996) 226-234.

[33] I. I. Aksenov, V. A. Belous, V. G. Padalka, and V. M. Khoroshikh, Sov. J. Plasma Phys. 4 (1978) 425-428.

[34] A. Anders, S. Anders, and I. G. Brown, Plasma Sources Sci. \& Technol. 4 (1995) 1-12.

[35] D. B. Boercker, D. M. Sanders, J. Storer, and S. Falabella, J. Appl. Phys. 69 (1991) 115120.

[36] C. A. Davis and I. J. Donelly, J. Appl. Phys. 72 (1992) 1740-1747.

[37] J. Storer, J. E. Galvin, and I. G. Brown, J. Appl. Phys. 66 (1989) 5245-5250.

[38] V. S. Veerasamy, G. A. J. Amaratunga, and W. I. Milne, IEEE Trans. Plasma Sci. 21 (1993) 322-328.

[39] M. M. M. Bilek, Y. Yin, and D. R. McKenzie, IEEE Trans. Plasma Sci. 24 (1996) 11651173.

[40] B. Alterkop, E. Gidalevich, S. Goldsmith, and R. L. Boxman, J. Phys. D: Appl. Phys. 29 (1996) 3032-3038.

[41] B. Alterkop, E. Gidalevich, S. Goldsmith, and R. L. Boxman, J. Phys. D: Appl. Phys. 31 (1998) 873-879.

[42] B. R. Cluggish, IEEE Trans. Plasma Sci. 26 (1998) 1645-1652. 
[43] J. R. Treglio, Magnetically filtered cathodic arc plasma apparatus, Patent US 5317235, May 31, 1994.

[44] I. I. Aksenov, V. A. Belous, V. G. Padalka, and V. M. Khoroshikh, Instrum. Exp. Tech. 21 (1978) 1416-1418.

[45] J. Vyskocil and J. Musil, J. Vac. Sci. Technol. A 10 (1992) 1740-1748.

[46] P. J. Martin, S. Falabella, and D. M. Karpov, “Coatings from the vacuum arc deposition,” in Handbook of Vacuum Arc Science and Technology, R. L. Boxman, P. J. Martin, and D. M. Sanders, Eds. Park Ridge: Noyes, 1995, pp. 367-551.

[47] P. J. Martin, A.Bendavid, and T. J. Kinder, IEEE Trans. Plasma Sci. 25 (1997) 675-679.

[48] R. L. Boxman and S. Goldsmith, IEEE Trans. Plasma Sci. 17 (1989) 705-712.

[49] V. N. Zhitomirsky, R. L. Boxman, and S. Goldsmith, Surf. Coat. Technol. 68/69 (1994) 146-151.

[50] S. Anders, A. Anders, and I. G. Brown, J. Appl. Phys. 75 (1994) 4895-4899.

[51] A. Anders, S. Anders, and I. G. Brown, J. Appl. Phys. 75 (1994) 4900-4905.

[52] M. M. M. Bilek, D. R. McKenzie, Y. Yin, M. U. Chhowalla, and W. I. Milne, IEEE Trans. Plasma Sci. 24 (1996) 1291-1298.

[53] D. A. Baldwin and S. Fallabella, "Deposition processes utilizing a new filtered cathodic arc source,” presented at Proc. of the 38th Annual Techn. Conf., Society of Vacuum Coaters, 1995, 309-316.

[54] T. Schülke, A. Anders, and P. Siemroth, IEEE Trans. Plasma Sci. 26 (1997) 660-664.

[55] T. Witke, T. Schülke, B. Schultrich, P. Siemroth, and J. Vetter, "Comparison of filtered high current pulsed arc deposition ( $\phi$-HCA) with conventional vacuum arc methods,” 
presented at Int. Conf. Plasma Surface Engineering, Garmisch-Patenkirchen, Germany, 1998,

[56] V. I. Gorokhovsky, Personal Communication, 1999.

[57] R. P. Welty, Rectangular vacuum-arc plasma source, Patent 5,480,527, Jan. 2, 1996.

[58] D. B. Boercker, S. Falabella, and D. M. Sanders, Surf. Coat. Technol. 53 (1992) 239-242.

[59] S. Falabella and D. M. Sanders, J. Vac. Sci. Technol. A 10 (1992) 394-397.

[60] R. P. Welty, "Linear magnetron arc cathode and macroparticle filter," presented at Int. Conf. Metal. Coat. \& Thin Films, San Diego, 1999, paper B1-5.

[61] I. I. Aksenov, V. A. Belous, and V. M. Khoroshikh, "Formation of filtered intense vacuum-arc plasma flows,” presented at XVIIth Int. Symp. Discharges and Electrical Insulation in Vacuum, Berkeley, CA, 1996, 895-899.

[62] G. V. Kljuchkov, V. G. Padalka, L. P. Sablev, and R. I. Stupka, Plasma arc apparatus for applying coatings by means of a consumable cathode, Patent US 4492845, Jan. 8, 1985.

[63] A. I. Ryabchikov and I. B. Stepanov, Rev. Sci. Instrum. 69 (1998) 810-812.

[64] A. I. Ryabchikov, I. B. Stepanov, S. V. Dektjarev, and O. V. Sergeev, Rev. Sci. Instrum. 69 (1998) 893-895.

[65] A. I. Ryabchikov, Surf. \& Coat. Technol. 96 (1997) 9-15.

[66] M. M. M. Bilek, A. Anders, and I. G. Brown, IEEE Trans. Plasma Sci. 27 (1999) in print.

[67] J. Koskinen, A. Anttila, and J.-P. Hirvonen, Surf. Coat. Technol. 47 (1991) 180-187.

[68] A. Anders, Surf. \& Coat. Technol. 93 (1998) 158-167.

[69] M. Harkovita, J. Salo, A. Anttila, and R. Lappalainen, Diamond and Rel. Mat. 4 (1995) 1335-1339. 
[70] S. Anders, A. Anders, M. R. Dickinson, R. A. MacGill, and I. G. Brown, IEEE Trans. Plasma Sci. 26 (1997) 670-674.

[71] M. M. M. Bilek and I. G. Brown, Rev. Sci. Instrum. 69 (1998) 3353-3356.

[72] C. F. Meyer and H.-J. Scheibe, "Filtered Laser-Arc: A new technology for deposition of particlefree films,” presented at Int. Conf. Metal. Coat. \& Thin Films (ICMCTF), San Diego, 1999, paper B4-9.

[73] T. Schülke and A. Anders, Plasma Sources Sci. Technol. 8 (1999) in print.

[74] M. Keidar, I. I. Beilis, R. Aharonov, D. Arbilly, R. L. Boxman, and S. Goldsmith, J. Phys. D: Appl Phys. 30 (1997) 2972-2978.

[75] M. Keidar, I. Beilis, R. L. Boxman, and S. Goldsmith, Surf. Coat. Technol. 86/87 (1996) $415-420$.

[76] R. Aharonov and M. Keidar, "Influence of an electric field on the macroparticle size distribution in a vacuum arc,” presented at Int. Conf. Metal. Coat. \& Thin Films, San Diego, 1999, paper B1-6.

[77] Y. Yin and D. R. McKenzie, J. Vac. Sci. Technol. A 14 (1996) 3059-3064. 